\title{
Acute Myocardial Infarction Immediately after Second Vaccination for Coronavirus Disease 2019
}

\author{
atsumasa kurozumi $^{1}$, hisao hara ${ }^{1}$, ran nagai ${ }^{1}$, and yukio hiroi ${ }^{1}$ \\ ${ }^{1}$ National Center for Global Health and Medicine
}

March 2, 2022

\begin{abstract}
We present a serious case of acute myocardial infarction soon after second vaccination for coronavirus disease 2019 . Her pathological findings and intravascular imaging partly unveil how vaccine can cause acute coronary syndrome. It also shows the importance to observe closely high-risk patients of cardiovascular diseases after vaccination.
\end{abstract}

\section{Title of Case}

Acute Myocardial Infarction Immediately after Second Vaccination for Coronavirus Disease 2019

Atsumasa Kurozumi, Hisao Hara, Ran Nagai, Yukio Hiroi

Department of Cardiology, National Center for Global Health and Medicine, Shinjuku-city, Tokyo, Japan

\section{A Key Clinical Message}

Acute myocardial infarction following vaccination for coronavirus disease 2019 has been rarely reported, but has shown severe cases. Physicians should cite them as a differential diagnosis, and properly treat patients with this rare condition.

\begin{abstract}
We present a serious case of acute myocardial infarction soon after second vaccination for coronavirus disease 2019. Her pathological findings and intravascular imaging partly unveil how vaccine can cause acute coronary syndrome. It also shows the importance to observe closely high-risk patients of cardiovascular diseases after vaccination.
\end{abstract}

\section{Background}

The current outbreak of the coronavirus disease 2019 (COVID-19) has caused many severe cases of pneumonia. A messenger RNA (mRNA) vaccine against the COVID-19 virus has been in use since December 2020 [1]. However, this vaccine has been reported to cause adverse events, including cardiovascular ones, and the underlying mechanisms of these adverse events are unknown [2,3,4]. Herein, we report a rare case of acute myocardial infarction shortly after COVID-19 vaccination and partially clarify the pathogenesis of the acute myocardial infarction.

\section{Case Presentation}

A 70-year-old female patient with hypertension, dyslipidaemia, diabetes mellitus, and no allergic history presented to our emergency care centre with nausea and chest pain $15 \mathrm{~min}$ after her second dose of the Pfizer-BioNTech COVID-19 vaccine. Physical examination revealed peripheral cyanosis and the following 
vital signs: heart rate, 57 beats/min; blood pressure, $84 / 67 \mathrm{mmHg}$; respiratory rate, 22 breaths/min; oxygen saturation, $97 \%$ in room air; and temperature, 35.6. Lung and cardiac auscultation findings were normal.

An initial electrocardiogram (Fig. 1) showed ST-segment elevation in II, III, and aVF and a first-degree atrioventricular block. Transthoracic echocardiograms showed inferior wall hypokinesia. Chest radiography revealed clear bilateral lung fields. Her cardiac enzymes including inflammatory markers (white blood cell count and $\mathrm{C}$ reactive protein level) were still normal, showing no acceleration of blood coagulation (D-dimer and fibrinogen). An urgent catheter examination (Fig. 2) showed total occlusion in the right coronary artery (RCA) \#3 and $90 \%$ stenosis in the left anterior descending artery (LAD) \#7.

After catheterisation, percutaneous coronary intervention was performed on the RCA lesion. A red thrombus was obtained by thrombectomy, and the occlusion was released. A new distal occlusion was found in the fourth posterolateral branch artery (\#4PL) and posterior descending artery (\#4PD) of the RCA (Fig. 3) with residual ST-segment elevation in II, III, and aVF. Intravascular ultrasonography (IVUS) did not identify any obvious plaque rupture whereas continuous diffuse plaques and some unstable like lesions were observed (Fig. 3). TIMI III blood flow was obtained, and there were large amount of blood clots and distal embolization. Therefore, we did not place a stent, but instead placed intra-aortic balloon pumping (IABP) and returned to the ward.

The next day, optical coherence tomography (OCT) was performed to evaluate the unstable vessel. On coronary angiography, the \#4PD/PL thrombus had disappeared (Fig. 4). Thrombus and vasa vasorum in the plaque layer (Fig. 4) were observed by OCT, indicating an unstable lesion. Therefore, we implanted a drug-eluting stent in the lesion where the vasa vasorum were present. IABP was discontinued after the intervention. During hospitalisation, the LAD lesion was treated using percutaneous coronary intervention. The patient was on medications, including high-dose statins, for the treatment of myocardial infarction and was recovering well; she was discharged on day 13 following the Japanese Circulation Society guideline. The thrombus contained atheroma with cholesterol crystals and foam cells (Fig. 5).

\section{Outcome and Follow-up}

On 6 months after discharge, she has experienced no symptoms related to cardiovascular disease, and no adverse and unanticipated events with her medication.

\section{Discussion}

Cardiovascular events have been reported as side effects of the COVID-19 vaccine; however, it is still unclear how vaccination can be associated with acute coronary syndrome (ACS) $[2,3,4]$, coronary embolism due to severe inflammation induced by the vaccine, or coronary thrombosis mainly caused by plaque rupture.

Coronary embolism is a rare disease and is difficult to distinguish from coronary thrombosis. It has been reported to be caused occasionally in patients with arrhythmia and valvular disease [5]. Moreover, inflammatory hypercoagulation is known as a mechanism of coronary embolism [6]. It is often diagnosed by relating these conditions to embolism and by features such as fewer atherosclerosis lesions on specific angiographic images [6]. The typical thrombus is found to be platelet and fibrin rich on pathological examination [6].

This patient had an atypical clinical history, leading to myocardial infarction shortly after vaccination. Therefore, we considered that the onset was related to the vaccine. It was difficult to find the culprit lesion even using IVUS and OCT. However, we suspected myocardial infarction caused by plaque rupture, rather than by emboli, from the results of the pathological examination of the collected thrombus, which contained atheroma rich in cholesterin crystals and foam cells.

The vasa vasorum are small vessels that provide blood to the walls of arteries and veins. It is known that atherosclerosis facilitates the growth of vulnerable plaques and promotes neovascularisation of the vasa vasorum, which predisposes plaques to rupture [7]. Treatment with a low dose of statin has a little effect on the vasa vasorum, and a high dose is often needed [8]. Other medications for this condition are not known to date. 
Through OCT, we discovered a rare large vasa vasorum in a thick plaque, indicating that it was unstable. These findings support the theory that plaque rupture led to the patient's ACS. Therefore, angioplasty was performed, and high-dose statins were administered for these lesions.

Only three cases of acute myocardial infarction have been reported to occur within 2 hours after COVID-19 vaccination $[2,3,4]$. Patients in all three cases had RCA lesions; this finding was similar to that in the present case report. Kounis syndrome has been reported to occur after the vaccination [9]. Around $70 \%$ of patients with Kounis syndrome show ST elevation in inferior leads on electrocardiography [10]; the reason for high probability of RCA lesion still has been unknown. It might also support the fact that acute myocardial infarct immediately after vaccination is caused by allergic factors like Kounis syndrome. The patient in the present case was at a high risk of ACS. We suspected that stress or an allergic reaction caused by the vaccination might have led to elevated blood pressure and vasoconstriction, which affected the vulnerable plaque and caused plaque rupture.

\section{Conclusion}

The case we encountered is extremely rare in four respects: ACS occurred immediately after vaccination, the collected thrombus was evaluated pathologically, it showed large vasa vasorum, and the unknown aetiology of COVID-19 vaccine-related ACS was partly clarified. Careful observation after vaccination is necessary for those at high risk of cardiovascular diseases, and intravascular imaging and pathological examination of the thrombus are effective for evaluating coronary lesions and clarifying the pathogenesis of ACS.

\section{Acknowledgements}

none

\section{Ethical Approval}

Hereby, I, Atsumasa Kurozumi, consciously assure that for the manuscript "Acute Myocardial Infarction Immediately after Second Vaccination for Coronavirus Disease 2019" the following is fulfilled: (1) This material is the authors' own original work, which has not been previously published elsewhere. (2) The paper is not currently being considered for publication elsewhere. (3) The paper reflects the authors' own research and analysis in a truthful and complete manner. (4) The results are appropriately placed in the context of prior and existing research. (5) All sources used are properly disclosed (correct citation). Literally copying of text must be indicated as such by using quotation marks and giving proper reference.

\section{Consent}

Hereby, I, Atsumasa Kurozumi, confirmed that patient consent has been signed and collected in accordance with the journal's patient consent policy. I confirm that the data supporting the findings of this study are available within the article and its Supplementary material. Raw data that support the findings of this study are available from the corresponding author, upon reasonable request. I confirm that This research received no external funding.

\section{Author Contributions}

Atsumasa Kurozumi: directly involved in patient care or have undertaken or interpreted patient investigation, and drafted the article or revised it critically for important intellectual content

Hisao Hara: drafted the article or revised it critically for important intellectual content

Ran Nagai: directly involved in patient care or have undertaken or interpreted patient investigation

Yukio Hiroi: drafted the article or revised it critically for important intellectual content

\section{References}

[1] Papini F, Grassi N, Guglielmi G, Gattini V, Rago L, Bisordi C, et al. Covid-19 vaccine management (Comirnaty and mrna-1273 Moderna) in a teaching hospital in Italy: a short report on the vaccination 
campaign. Environ Health Prev Med. 2021;26:99.

[2] Maadarani O, Bitar Z, Elzoueiry M, Nader M, Abdelfatah M, Zaalouk T, et al. Myocardial infarction post COVID-19 vaccine - coincidence, Kounis syndrome or other explanation - time will tell. JRSM Open 2021;12:1-3.

[3] Tajstra M, Jaroszewicz J, Gasior M. Acute Coronary Tree Thrombosis After Vaccination for COVID-19. JACC Cardiovasc Interv 2021;14:e103-e104.

[4] Boivin Z, Martin J. Untimely Myocardial Infarction or COVID-19 Vaccine Side Effect. Cureus 2021;13:e13651.

[5] Lacey MJ, Raza S, Rehman H, Puri R, Bhatt DL, Kalra A. Coronary Embolism: a systematic review. Cardiovasc Revasc Med 2020;21:367-374.

[6] Raphael CE, Heit JA, Reeder GS, Bois MC, Maleszewski JJ, Tilbury RT, et al. Coronary embolus: an underappreciated cause of acute coronary syndromes. J Am Coll Cardiol Intv 2018;11:172-180.

[7] Xu J, Lu X, Shi G-P. Vasa vasorum in atherosclerosis and clinical significance. Int J Mol Sci 2015;16:1157411608.

[8] Urbich C, Dernbach E, Zeiher AM, Dimmeler S. Double-edged role of statins in angiogenesis signaling. Circ Res 2002: 90: 737-744.

[9] Özdemir IH, Özlek B, Özen MB, Gündüz R, Bayturan Ö. Type 1 Kounis syndrome induced by inactivated SARS-COV-2 vaccine. J Emerg Med https://doi.org/10.1016/j.jemermed.2021.04.018 (14 November 2021)

[10] Abdelghany M, Subedi R, Shah S, Kozman H. Kounis syndrome: A review article on epidemiology, diagnostic findings, management, and complications of allergic acute coronary syndrome. Int J Cardiol $2017 ; 232: 1-4$.

\section{Figure Legends}

Fig. 1.

Electrocardiography shows ST-segment elevation in II, III, and aVF and a first-degree atrioventricular block.

Fig. 2 .

Urgent catheter examination shows total occlusion in the right coronary artery (RCA) \#3 and 90\% stenosis in the left anterior descending (LAD) artery \#7.

Fig. 3 .

The distal occlusion in the RCA the fourth posterolateral branch artery (\#4PL) and posterior descending artery (\#4PD) was newly found after thrombectomy.

Intravascular ultrasound likely shows thick plaques [white arrows] and thin fibrous capsules on lipid pool plaques [red arrows].

Fig. 4 .

The follow-up coronary angiography is normal, including RCA \#4PD/PL findings.

Optical coherence tomography shows white thrombus [white arrow] and a large vasa vasorum in the plaque layer near the thrombus [red arrows].

Fig. 5 .

The thrombus contains atheroma rich in cholesterin crystal [black arrow] and foam cells [white arrow]. 

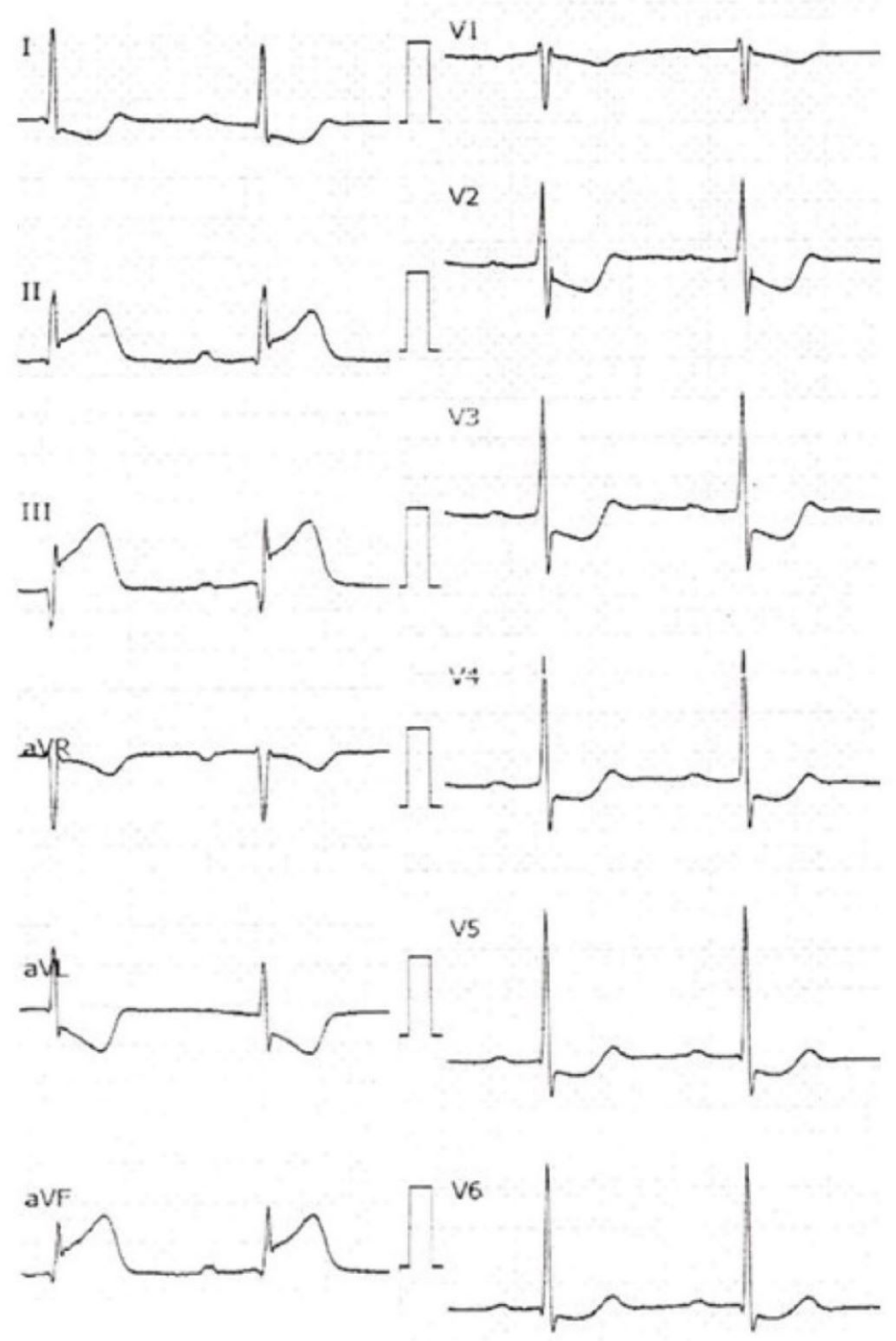

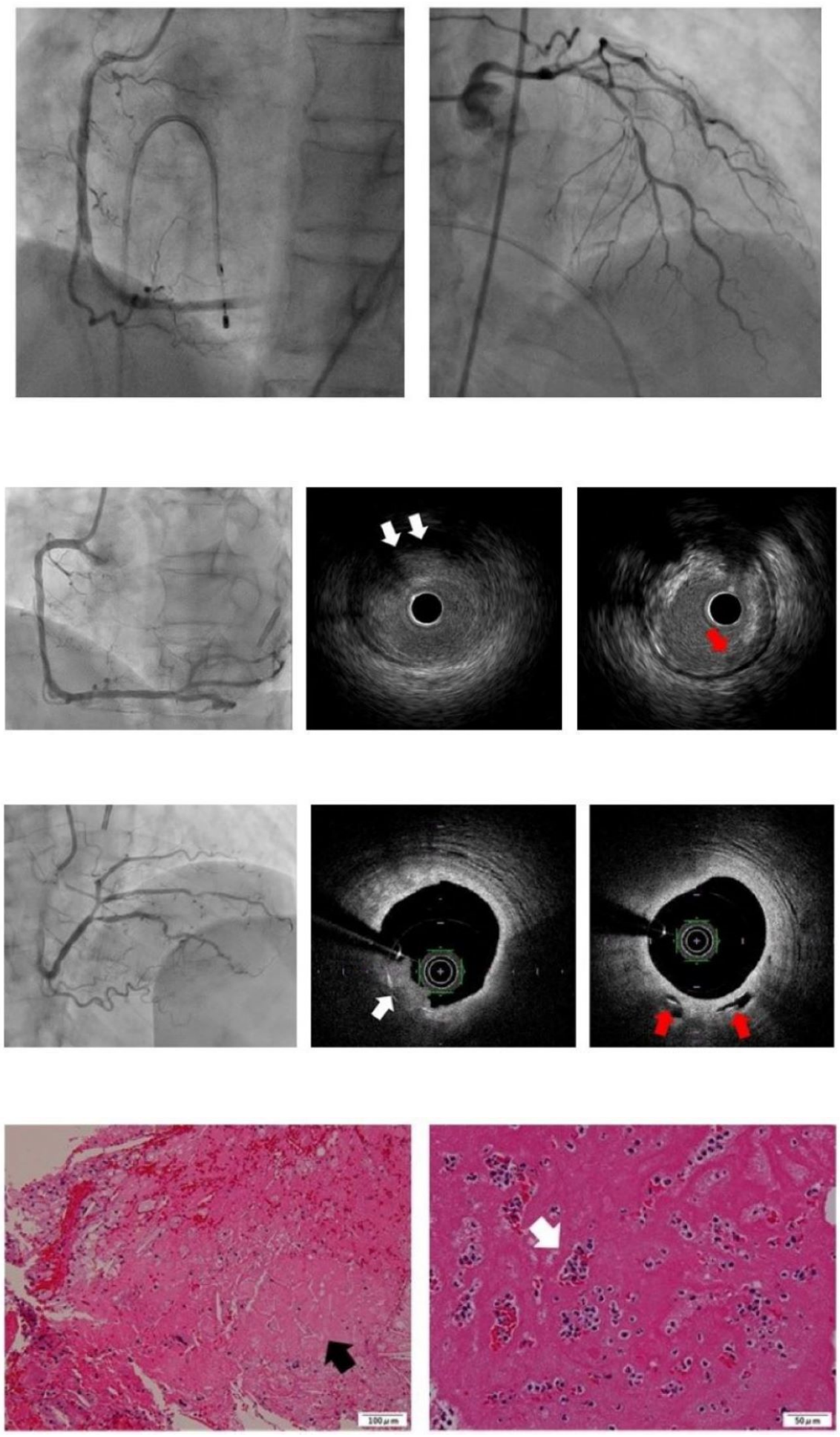